\title{
EVALUACIÓN DEL DISCURSO NARRATIVO EN PACIENTES CON DAÑO CEREBRAL LEVE
}

\section{EVALUATION OF THE NARRATIVE SPEECH IN PATIENTS WITH MILD CEREBRAL INJURY}

\author{
ANA PÉREZ CACHÓN \\ Unidad de Daño Cerebral. Hospital Beata María Ana. C/ Vaquerías, s/n. 28007 Madrid \\ e-mail: anapecacho@hotmail.com
}

\section{Resumen}

En la práctica clínica de la rehabilitación del lenguaje los terapeutas disponen de pocas pruebas que puedan evaluar el discurso oral. Las baterías para la valoración de las afasias que se emplean habitualmente no suelen aportan datos de este tipo, cuando lo hacen el resultado son escalas que describen globalmente el lenguaje del sujeto y que no posibilitan un seguimiento pormenorizado de la evolución. La importancia de valorar la producción en el nivel de discurso se hace más evidente en el estudio de pacientes con daños leves o en un momento avanzado de recuperación que presentan una competencia alta en muchos casos no muestran déficit de lenguaje en tareas más sencillas. Una valoración que obvia el discurso tiende a producir una sobreestimación de las capacidades de estos sujetos y con frecuencia deriva en una intervención inadecuada e incompleta. El propósito del presente trabajo es hacer una revisión de estudios que abordan el análisis del discurso narrativo en daño cerebral y explorar, a través de un caso, algunas aplicaciones de este tipo de estudios en la evaluación del lenguaje de sujetos con afectación leve.

\section{Palabras Clave}

lenguaje, discurso, evaluación, daño cerebral leve.

\begin{abstract}
In clinical practice there are few test that language therapist can use for the oral speech evaluation. The battery that has been frecuently used for the aphasia assessment, doesn't usually give us this type of data, and when it happens, it is just a global description of the language of the subject, which doesn't make possible a detailed pursuit of the evolution. The relevance of linguistic production's assessment becomes more evident in the study of patients with mild injury as well as when patient is in an advanced recovery stage. An assessment without a speech analysis uses to overestimate the subject's capacities and usually ends in an incomplete and inadequate intervention. The aim of the present work was to review different studies that include the narrative speech as part of the assessment, and to explore, with the study of a a single case, some applications in this kind of studies for language evaluation in mild brain injury patients.
\end{abstract}

\section{Key Words}

Language, speech, assessment, mild brain injury. 


\section{Introducción}

La rehabilitación de personas que ha sufrido un Daño Cerebral tiene como meta contribuir a la mejora del funcionamiento de estos sujetos en la vida cotidiana. Esto requiere que la intervención se estructure en torno a objetivos de carácter funcional y por ello cada vez más las valoraciones tienen que ser capaces de detectar las necesidades de tratamiento de los pacientes y de determinar el grado de logro de los objetivos de intervención (Muñoz Céspedes y Tirapu, 2001).

En la practica clínica con cierta frecuencia se ha de abordar el trabajo con personas que, bien por el carácter leve de la lesión inicial, bien por encontrarse en estadios avanzados de la rehabilitación, alcanzan un nivel de competencia alto en las tareas de lenguaje que se les presentan como parte de la evaluación. Estos sujetos pueden mostrar sistemáticamente niveles de techo en estas pruebas y al mismo tiempo manifestar dificultades para mantener una conversación, relatar coherentemente un suceso o hacer una descripción.

Obviar una valoración específica del lenguaje a través de la conversación o de narraciones tiende a producir una sobrestimación de la capacidad lingüística de estos sujetos (Coelho, Liles y Duffy, 1991) que deriva en una definición inadecuada de los objetivos de intervención. Esto resulta más grave si se tiene en cuenta que precisamente estas tareas son parte de las demandas reales que impone la vida cotidiana. Conversar con alguien, contar una historia, describir un lugar o hacer planes es evidentemente mucho más funcional que leer palabras que no existen o repetir frases de longitud creciente.

Por esto una intervención que quiera orientar sus esfuerzos a la mejora de competencias funcionales del paciente debe incluir la conversación y el discurso como elementos destacados dentro de la valoración del lenguaje.

\section{Material y método}

La evaluación en la practica clínica esta ineludiblemente ligada a la intervención, su propósito no es sólo describir la situación de parti- da del sujeto sino que debe resultar útil en la definición de los objetivos de la intervención y en la medición del grado de logro de los mismos. Esto implica que no existe un único modo de valorar y que es labor de los terapeutas seleccionar en cada ocasión aquellos instrumentos que se adecuan mejor a estos fines. De modo general un instrumento de evaluación se considera útil si es capaz de:

- Adaptar los contenidos y el grado de dificultad al nivel del sujeto.

- Posibilitar la detección de los mecanismo cognitivos alterados.

- Mostrar validez ecológica.

- Ser sensible a los cambios en los niveles de ejecución del sujeto a lo largo del tiempo.

(Muñoz Céspedes y Tirapu, 2001).

En las baterías que se vienen aplicando para la valoración de las afasias (Valle y Cuetos, 1995; Ducarne de Ribacourt,1976; Peña- Casanova, 1990; García Albea, Sánchez Bernardos y del Viso, 1996) no siempre se pueden encontrar apartados o subapartados que valoren el discurso oral. En los casos en que existen, se trata de escalas de severidad en las que se puntúa el grado afectación en varios parámetros dando lugar a perfiles (Peña- Casanova, 1990; García Albea, Sánchez Bernardos y del Viso, 1996) o bien, de escalas descriptivas del tipo de producción del paciente (Peña- Casanova, 1990; García Albea, Sánchez Bernardos y del Viso, 1996) a través de las que se intenta caracterizar el habla y el discurso. Tras una revisión de las pruebas utilizadas y de las medidas resultantes (vid. anexo 1) se puede concluir que resultan insuficientes para la valoración del discurso no sólo por la escasez de ítems orientados a este fin sino fundamentalmente por el tipo de información que aportan. Los datos que se obtienen son apreciaciones subjetivas y amplias que no contribuyen a la formulación de hipótesis sobre el modo en que está alterado el discurso ni son capaces de medir parámetros que posteriormente resulten útiles para un seguimiento.

Afortunadamente la evaluación del lenguaje trasciende la administración de baterías y pruebas estandarizadas que, si bien constituyen una primera y necesaria aproximación al caso, rara 
vez resultan suficientes para cubrir los objetivos de una evaluación. (Solberg y Mateer cit. Coelho, Liles y Duffy, 1991). Desde hace unos años los investigadores han encontrado en el análisis del discurso (AD) un instrumento útil para estudiar la alteración de la competencia lingüística en los pacientes con daño cerebral.

En esta metodología se parte de una muestra de discurso que es analizado a través de la medición de una serie de parámetros de lenguaje a diferentes niveles. Habitualmente se solicita al paciente que haga una descripción de un suceso o que cuente una pequeña historia que idealmente debe tener una longitud de cinco frases o más (Coelho, Liles y Duffy, 1991) mientras se registra en audio o video la producción resultante. Posteriormente la muestra recogida se transcribe y sobre la trascripción se analizan elementos relativos a la macro y a la micro estructura del texto.

Los modos de producción de la muestra que más frecuentemente se han empleado en los estudios son la descripción de los sucesos en un dibujo (Joanette y Goulet, 1990; Galsky, Tompkins y Johnston,1998) y el relato de una historia reflejada en una serie de viñetas. (Galsky, Tompkins y Johnston,1998; Tucker y Hanlon, 1998; Ulatowska, Allar y Bond, 1990). Otras formas de obtener un discurso narrativo son pedir el relato de una historia previamente escuchada (Ulatowska, Allar y Bond, 1990; Body y Perkins, $1998,2004)$, hacer un relato libre sobre una experiencia personal destacada (Ulatowska, Allar y Bond, 1990) o resumir una historia previamente relatada (Ulatowska, Allar y Bond, 1990). En otras ocasiones los autores emplean entrevistas semi-estructuradas con temáticas sencillas como explicar un día en su vida cotidiana del paciente (Ellis et al. 2005). Cuando se trata de obtener un discurso procedimental se demanda una explicación sobre el modo de llevar a cabo una rutina como puede ser lavarse los dientes o cortar el pan, o algunas otras tareas más específicas y complejas como cambiar la rueda de un coche (Ulatowska, Allar y Bond, 1990). Con menos frecuencia se busca la producción de descripciones o se recogen muestras conversacionales y en muchos casos se combinan varios tipos de discurso en un mismo estudio (Coelho, 2003).
Hay que destacar que tanto el tipo de marcadores textuales como la macro estructura resultante varían en función de la naturaleza del tipo de discurso que se elicita (narrativo, procedimental, descriptivo o conversacional) por lo que la elección de uno u otro tipo no es inocua (Coelho, 2003).

De igual modo la tarea de elicitación empleada influye en las respuestas que se obtienen incluso cuando el tipo de discurso es el mismo. Así, comparando narraciones libres y elicitadas mediante el recuento de historias Liles et al. encuentran respuesta consistentes en las que se aprecia un incremento de la complejidad sintáctica en narraciones libres y un aumento de unidades de contenido en la reproducción de una historia previamente escuchada, tanto en el caso de sujetos con daño cerebral como en controles (Liles et. al, cit en Coelho 2003).

Una vez obtenida y transcrita la muestra los parámetros que se miden en cada caso dependen del objeto de estudio dentro del discurso y abarcan desde aspecto léxicos y sintácticos hasta la estructura misma del texto (Joanette y Goulet, 1990).

En la literatura los autores proponen diversos índices y recuentos para sus estudios que de forma general, y atendiendo a nivel de análisis en el que inciden, se pueden sudividir en dos categorías: parámetros de nivel de frase o microestructurales y parámetros de nivel de discurso o macroestructruales.

Los análisis de la micro estructura se orientan a realizar medidas sobre aspectos léxicos y análisis sintácticos dentro de la misma frase.

Los primeros usualmente son recuentos y «ratios» de relación, bien entre elementos de diferentes categorías sintácticas (porcentaje nombre / verbo, ratio adjetivo / nombre...), o bien entre alguna de ellas y el contenido vehiculado por la narración (ratio pronombre / concepto) (Joanette y Goulet, 1990).

El análisis a nivel sintáctico se realiza a través del estudio de la complejidad y/o de la eficiencia del lenguaje. Los índices que se toman como medida de la complejidad se relacionan frecuentemente con la aparición de oraciones subordinadas en el discurso. Una medida 
ampliamente aplicada es el porcentaje de oraciones subordinadas sobre el total (Joanette y Goulet, 1990) y otros modos de medirla son hallar la media del número de palabras dentro de una unidad de contenido (Galsky, Tompkins y Johnston,1998; Ulatowska, Allar y Bond, 1990), contabilizar el número de cláusulas dentro de otras unidades (Ulatowska, Allar y Bond, 1990) y cuantificar la presencia de conectores gramaticales (Ulatowska, Allar y Bond, 1990). Valorar la eficiencia supone conocer la relación entre la cantidad y el contenido en el texto, medida que puede concretarse en el recuento del número de palabras o de unidades formales que se requieren para la transmisión de un contenido (Galsky, Tompkins y Johnston,1998; Peña- Casanova 1995).

Sobre la misma muestra de discurso se suele realizar un análisis macroestructural. Éste hace referencia a las interrelaciones entre frases y busca la estructura textual subyacente en la producción del paciente. En este nivel algunos autores fijan como objeto de estudio la cohesión sintáctica y la coherencia semántica de la narración. Para cuantificarlas se usan medidas como el ratio pronombre/ nombre o sintagma nominal (Body y Perkins, 2004), en el caso de la cohesión, que informa sobre la capacidad del sujeto para explicitar el referente en un discurso; mientras para medir la coherencia es necesario identificar, clasificar y juzgar la adecuación de marcadores de cohesión, generalmente nexos inter oracionales (Ellis et al. 2005).

El estudio de la producción a nivel textual puede pasar por «traducir» en sus proposiciones el discurso para posteriormente valorar la cantidad y la naturaleza de la información y hacer emerger las relaciones lógicas entre los elementos del relato (Joanette y Goulet, 1990). En otras ocasiones se establecen a priori las unidades de significado que esperan elicitar con un grupo de estímulos y se puntúa la ejecución del sujeto en función del número de unidades de contenido que finalmente produce, del ajuste de la historia relatada al estímulo y de la aparición de elementos de contenido implícito que se esperaba elicitiar (Tucker y Hanlon, 1998). En esta misma línea de predefinir elementos elicitables, hay quien parte de las producciones de sujetos normales para determinar un grupo de proposi- ciones "canónicas» y poder medir luego su frecuencia de aparición y la co-ocurrencia de unas con otras en sujetos patológicos (Joanette y Goulet, 1990).

El abanico de parámetros medidos sobre la base de una muestra de habla es amplísimo. (Para una revisión se puede consultar el anexo 2 en el que se ofrece una clasificación de los que aparecen en estudios Coelho 2003; Joannete y Goulet, 1990 y Ulatowsta et al. 1990 que abordan el análisis desde diferentes enfoques y Coelho 1991 que recoge y resume otros tanto trabajos previos.)

La elección de unos parámetros u otros responde al interés del investigador en cada momento. este tipo de estudios presenta la ventaja de que una vez hecho el registro y la transcripción se pueden aplicar análisis sucesivos a diferentes niveles.

A pesar de las oportunidades que ofrece el $\mathrm{AD}$ existen algunas criticas que acompañan a los estudios sobre lenguaje en uso (Nickels, 2003) entre las que destacan:

1. La existencia de un alto grado de variabilidad entre hablantes no sólo cuantitativamente sino también cualitativamente, lo que dificulta determinar dónde esta la normalidad.

2. Problemas para medir las variaciones a lo largo del tiempo debido a las diferencias intra individuales en diferentes registros.

Parece por ello que las medidas cualitativas intra sujeto son más consistentes y algunos investigadores (Perkins et al. cit Nickels, 2003) recomiendan el uso de estas para conocer los resultados de los tratamientos. En este mismo sentido parece lógico tender al uso de parámetros que no dependan de la longitud del texto, es decir, de aquellos que relacionan unos elementos con otros dentro del mismo texto (porcentaje de subordinadas sobre el total mejor que número de subordinadas en una producción) y que permitan la comparación entre sucesivas producciones.

\section{Aplicación clínica}

Desde la practica clínica la aplicación de este tipo de análisis a la valoración tiene como obje- 
tivo hacer diagnósticos más precisos y posibilitar un seguimiento de los avances que se producen a lo largo de la rehabilitación. Consecuentemente se trata de estudios intrasujeto en los que la comparación cualitativa y cuantitativa de los resultados en sucesivos registros son tomados como medida de evolución y en los que estos resultados se combinan con otros procedentes de tareas clásicas y con las observaciones del propio terapeuta para valorar su significatividad. A continuación se presenta un caso en el que el $\mathrm{AD}$ se empleó como medio para medir la evolución de la anomia, las difluencias en el habla y la eficacia léxica a lo largo de la intervención, mostrándose eficaz a tal efecto.

\section{Presentación del caso}

Paciente varón de 57 años, diestro con estudios de universitarios en periodismo e historia moderna, político en activo, sufre un ACV como consecuencia de hipertensión arterial, a penas un mes después ingresa en nuestra Unidad. La imagen de resonancia magnética muestra lesión en el núcleo lenticular izquierdo. La exploración clínica del lenguaje evidencia intención comunicativa preservada y capacidad para mantener conversaciones con conciencia de sus dificultades. Su lenguaje es fluente con tendencia al agramatismo y la presencia de neologismos hace el discurso prácticamente ininteligible en tramos. La compresión de palabras y frases también está alterada mientras en el habla presenta una disartria leve. La evolución es muy positiva y dos meses más tarde puede mantener conversaciones con algunas dificultades de carácter anómico y una prosodia alterada. En ese momento sus resultados en el Boston Namig Test (García Albea et al. 1996) están dentro de la normalidad estadística con cierta lentitud en la ejecución. Es entonces cuando se empieza a aplicar el AD como modo de valoración del caso.

\section{Metodología}

Tras recoger una muestra de discurso elicitado a través de la tarea clásica de presentación de una lámina y transcribirla, se decide estu- diar tres aspectos que a su juicio están alterados:

- fluidez del habla

- fluidez del discurso

- eficacia de la narración

Posteriormente se buscan parámetros capaces de dar cuenta de estos aspectos y se adapta un protocolo de medición del discurso para el estudio de este y otros casos similares. Se toman como referencia tres de los parámetros del Perfil de Eficiencia Comunicativa (15): número total de palabras, número de unidades de contenido correctas y eficiencia léxica, se redefinen y se añade la ratio nombre/ verbo como medida de seguimiento de la anomia. Para el estudio de la fluidez del habla se emplean parámetros habituales de estudios del habla como conducta motora: velocidad de habla, bloqueos, alargamientos etc y para medir la adecuación del discurso se crea el índice que establece la relación entre las unidades de contenido correctas (que corresponden a la lámina y a la tarea solicitada) y el total del contenido.

El resultado son 12 parámetros y tres tipos de medidas:

Parámetros para medir el habla entendida como conducta motora:

- velocidad de habla

- bloqueos

- alargamientos

Parámetros que se relacionan con la generación y la organización del discurso:

- falsos inicios

- autocorrecciones

- repeticiones de palabras

- latiguillos

- silencios

- ratio nombre /verbo

Parámetros que hacen referencia la concreción del lenguaje o a la eficacia del mismo

- eficiencia léxica

- índice de adecuación

Para una definición detallada de estas medidas véase anexo 3. 
Estas variables fueron medidas soe discurso narrativo obtenido a través de la descripción de lámina tomada del Test de Boston (García Albea, Sánchez Bernardos y del Viso, 1996) y del Test de Barcelona (Peña- Casanova, 1990;).

\section{Resultados}

Los resultados son los se pueden observar en la tabla que sigue:

\begin{tabular}{|l|c|c|c|c|c|c|}
\hline \multirow{2}{*}{} & \multicolumn{2}{|c|}{ Inicial } & \multicolumn{2}{c|}{ 6 meses } & \multicolumn{2}{c|}{12 meses } \\
\cline { 2 - 7 } & 7 mar & $\%$ & 5 sep & $\%$ & 21 mar & $\%$ \\
\hline Número de palabras & 437 & - & 228 & - & 215 & - \\
\hline Número total de elementos & 457 & - & 231 & - & 219 & - \\
\hline Velocidad de habla & 90.41 & - & 116.41 & - & 107.50 & - \\
\hline Bloqueos & 25 & 5.47 & 3 & 1.31 & 6 & 2.79 \\
\hline Alargamiento de sonido & 36 & 7.87 & 3 & 1.31 & 15 & 5.37 \\
\hline Falsos inicios & 5 & 1.09 & 2 & 0.87 & 1 & 0.36 \\
\hline Autocorrecciones & 12 & 2.62 & 1 & 0.43 & 6 & 2.79 \\
\hline Repeticiones de palabras & 44 & 9.62 & 3 & 1.31 & 1 & 0.36 \\
\hline Latiguillos & 35 & 7.43 & 4 & 1.75 & 4 & 1.86 \\
\hline Silencios & 10 & 2.18 & 3 & 1.31 & 1 & 0.36 \\
\hline Ratio nombre / verbo & $53 / 46$ & 1.15 & $46 / 30$ & 1.53 & $43 / 19$ & 1.63 \\
\hline Eficiencia léxica & 2.06 & - & 1.94 & - & 2.02 & - \\
\hline Índice de adecuación & 46 & - & 88 & - & 92 & - \\
\hline
\end{tabular}

A través de los índices se puede apreciar la mejora que experimenta el discurso y que afecta especialmente a:

- la evocación de nombres, que aumenta en relación con la evocación de verbos.

- el ajuste del discurso a la demanda y la contenido que debía elicitarse por medio de la lámina presentada, medida por el índice de adecuación

- la mejora en la planificación y/o monitorización de su producción que se traduce en un descenso de los falsos inicios, de las repeticiones de palabras y el uso de latiguillos.

El análisis del discurso en el estudio de este paciente resulta de utilidad para:

- Cuantificar la aparición de la anomia en el discurso narrativo.

- Continuar midiendo la evolución de la anomia del paciente una vez que en tareas tradicionales de denominación por confrontación obtiene niveles de techo.

- Medir la alteración de la fluidez del habla: disponer de un línea base y de medidas de seguimiento y poder valorar su gravedad.

- Cuantificar el ajuste del discurso a la demanda hecha y apreciar la mejora que se produce a lo largo de la intervención.

- Cuantificar el déficit en la planificación de la frase y encontrar medidas para su seguimiento.

\section{Conclusiones}

- Las baterías para la valoración de las afasias no se muestran sensibles a las alteraciones de lenguaje que presentan sujetos con daños leves o en fases avanzadas de recuperación. 
- Frente a esto, el análisis del discurso se presenta como una metodología capaz de valorar a estos sujetos, respondiendo ampliamente a los criterios que hacen de una prueba de lenguaje un instrumento útil para orientar el proceso rehabilitador:

1. Adecua los contenidos y el grado de dificultad al nivel del paciente ya que parte de una muestra de habla elicitada y no requiere en este sentido un nivel específico previo para ser abordada.

2. Puede contribuir a la detección de los mecanismos alterados en la producción del lenguaje al permitir diferentes niveles de análisis (léxico, sintáctico, semántico, estructural...) del discurso generado.

3. Se le puede atribuir mayor validez ecológica que a las tareas específicas de lenguaje habituales en una evaluación (repetición, denominación, fluidez verbal), dado que, por un lado parte de muestras de lenguaje en uso que requieren el trabajo del sistema a varios niveles (estructuración del discurso, coherencia semántica, cohesión sintáctica, recuperación léxica...) por otro, es más cercano a las demandas de lenguaje a las que se enfrenta una persona en su vida cotidiana.

4. Muestra sensibilidad al cambio incluso en sujetos en los que la cercanía a la normalidad es alta y, en el caso que se presenta, ha permitido disponer de medidas cuantitativas para la valoración de la anomia en un momento de la evolución del paciente en que las tareas de denominación por confrontación no aportan más datos.

- El AD narrativo es factible en la practica clínica si bien su aplicación presenta algunas dificultades entre las que destacan la complejidad que supone decidir y delimitar las unidades de estudio, el coste temporal alto de este tipo de valoraciones y la ausencia de parámetros de estabilidad.

- Para seguir avanzando en la aplicación del $\mathrm{AD}$ como parte de la evaluación del len- guaje seria necesario disponer de estudios que detecten la estabilidad de los parámetros a lo largo de diferentes narraciones en un mismo sujeto puesto que conocer qué parámetros resultan estables intrasujeto permitiría usarlos como referencia en los seguimientos.

\section{Bibliografía}

Body, R. y Perkins, M. R. Ecological validity in assessment of discourse in traumatic brain injury: ratings by clinicians and non-clinicians. Brain injury, 1998, vol. 12, no. 11. 963-976.

Body, R. y Perkins, M. R. Validation of linguistic analyses in narrative discourse after traumatic brain injury. Brain injury, 2004, vol. 18. no 7, 707724.

Coelho, A., Liles, B. y Duffy, R. The use of discourse analices for the evaluation of higer level traumatically brain- injured adults. Brain Injury, 1991, vol. 5 n. ${ }^{\circ} 4,381-392$.

Ducarne de Ribacourt. Test para el examen de la afasia. TEA. Madrid: 1976.

Ellis, Ch., Rosenbeck, J. C.,Rittman, M.R. y Boylstein, C. A. Recovery of cohesion in narrative discourse after left-hemisfere stroke. Journal of rehabilitation research and development. 2005. vol. 42, no. 6. 737-746.

Galski, T., Tompkins, C. Y Johnston, M.V. Competence in discourse as a measure of social integration and quality of life in persons with traumatic brain injury, Brain injury, 1998, vol. 12, no 9. 769-782.

García Albea, J.E., Sánchez Bernardos, M.L. y del Viso, S. Test de Boston para el diagnóstico de la afasia: adaptación española. Editorial médica panamericana. Madrid. 1996.

Joanette, Y. Y Goulet, P., Narrative discourse in RightBrain-Damaged; Discourse ability and brain damage. Springer-Verlag, New York, 1990, 132.

Muñoz Céspedes y Tirapu, Evaluación neuropsicológica; Rehabilitación neuropsicológica, Síntesis, Madrid, 2001, 69.

Nickels, L. Tried, tested and trusted? Language assessment for rehabilitation. The effectiveness of rehabilitation for cognitive deficits. Oxford University press. Oxford. 2003. 
Peña Casanova, J. y Manero, R.M. El perfil de eficiencia comunicativa: control evolutivo y de la terapia. Rehabilitación de la afasia y trastornos asociados. Masson. Barcelona. 1995.

Peña-Casanova, J. Programa Integrado de Evaluación Neuropsicológica Barcelona. Masson. Barcelona.1990.

Tucker,F.M. y Hanlon, R.E., Effects of mild traumatic brain injury on narrative discourse production, Brain injury, 1998, vol. 12, no. 9, 783-792.
Ulatowska, H. K., Allard, L. y Bond Chapman, S. Narrative and procedural discourse in aphasia. Discourse ability and brain damage. Springer-Verlag, New York, 1990,182.

Valle, F y Cuetos, F. Evaluación del Procesamiento Lingüístico en la Afasia. Lawrence Erlbaum Associates. Londres. 1995.

\section{Anexo 1. Resumen de la revisión de las pruebas más habituales para la valoración de las afasias y el lenguaje}

\begin{tabular}{|c|c|c|}
\hline & $\begin{array}{l}\text { Elaboración del } \\
\text { discurso }\end{array}$ & Notas \\
\hline $\begin{array}{l}\text { Test para el examen } \\
\text { de la afasia. } \\
\text { (Ducarne de } \\
\text { Ribacourt) }\end{array}$ & $\begin{array}{l}\text { Descripción de } \\
\text { imágenes } \\
\text { Definición de } \\
\text { palabras } \\
\text { Explicación de } \\
\text { refranes }\end{array}$ & $\begin{array}{l}\text { DESCRIPCIÓN DE IMÁGENES } \\
\text { Se pide la descripción de varias láminas y se observa el nivel de } \\
\text { la descripción tomando como base los niveles de Binet:: enume- } \\
\text { ración, descripción e interpretación. } \\
\text { DEFINICIÓN DE PALABRAS Y ELABORACIÓN DE REFRANES } \\
\text { Se solicitan las tareas correspondientes y se valora según unas es- } \\
\text { calas descriptivas de la ejecución compuestas por tres y cuatro ni- } \\
\text { veles respectivamente de elaboración } \\
\text { Se hace una análisis cuantitativo, a través de los porcentajes de } \\
\text { acierto en cada prueba del test y uno cualitativo, por medio de es- } \\
\text { calas descriptivas para varios aspectos: } \\
\text { - pérdida de elementos lingüísticos } \\
\text { - defecto de evocación léxica } \\
\text { - falta de evocación sintáctica. }\end{array}$ \\
\hline $\begin{array}{c}\text { Test de Boston } \\
\text { para el diagnóstico } \\
\text { de la afasia: } \\
\text { adaptación española. }\end{array}$ & $\begin{array}{c}\text { Perfil de } \\
\text { características } \\
\text { del habla }\end{array}$ & $\begin{array}{l}\text { PERFIL DE CARACTERÍSTICAS DEL HABLA } \\
\text { Valora seis características del habla que, afirma, no pueden me- } \\
\text { dirse objetivamente de modo satisfactorio, por lo que propone una } \\
\text { escala de severidad de } 1 \text { a } 7 \text { (excepto para la escala de encontrar } \\
\text { palabras). Los parámetros estudiados son: línea melódica, longitud } \\
\text { de la frase, agilidad articulatoria, forma gramatical, parafasia en } \\
\text { habla seguida y capacidad para encontrar palabras. }\end{array}$ \\
\hline
\end{tabular}


Anexo 1. (Continuación)

\begin{tabular}{|c|c|c|}
\hline & $\begin{array}{l}\text { Elaboración del } \\
\text { discurso }\end{array}$ & Notas \\
\hline $\begin{array}{l}\text { Programa Integrado } \\
\text { de Evaluación } \\
\text { Neuropsicológica Bar- } \\
\text { celona. }\end{array}$ & $\begin{array}{l}\text { Narración } \\
\text { temática. } \\
\text { Fluencia verbal } \\
\text { y contenido } \\
\text { informativo }\end{array}$ & $\begin{array}{l}\text { NARRACIÓN TEMÁTICA } \\
\text { Escala descriptiva de } 0 \text { a } 6 \text { puntos y que mide sobre todo fluencia } \\
\text { (en el sentido clásico en afasiolgía). } \\
\text { 0. No comunicación } \\
\text { 1. Lenguaje no fluente. Palabras aisladas. } \\
\text { 2. Lenguaje no fluente. Agramatical. } \\
\text { 3. Lenguaje fluente tipo afásico. } \\
\text { 4. Lenguaje cualitativamente no afásico. } \\
\text { 5. Lenguaje fluente no afásico (anomia discreta o circunlo- } \\
\text { quios). } \\
\text { 6. Lenguaje normal. } \\
\text { FLUENCIA VERBAL } \\
\text { Valora en una escala el lenguaje del paciente partiendo de mues- } \\
\text { tras en conversación, descripción y narración. Sigue la normativa } \\
\text { de Kertestz (1979) dividiendo la escala de fluencia en } 11 \text { grados, } \\
\text { mide fundamentalmente: presencia de automatismos, organización } \\
\text { gramatical, aparición de jerga y circunloquios, anomia y longitud } \\
\text { y complejidad de frase. } \\
\text { ConteNIDO INFORMATIVO } \\
\text { Metodología similar en } 11 \text { grados de } 0 \text { a } 10 \text { mide el numero de } \\
\text { respuestas correctas a una serie de preguntas en conversación. }\end{array}$ \\
\hline $\begin{array}{l}\text { Evaluación del } \\
\text { Procesamiento } \\
\text { Lingüístico } \\
\text { en la Afasia. }\end{array}$ & $\begin{array}{l}\text { No dispone de } \\
\text { ninguna prueba } \\
\text { que valore } \\
\text { discurso. }\end{array}$ & \\
\hline
\end{tabular}




\section{Anexo 2. Tabla resumen de los parámetros utilizados en ad en estudios sobre daño cerebral}

Presenta un resumen de los parámetros empleados en cuatro de los estudios revisados (Coelho 1991, 2003; Joannete y Goulet, 1990 y Ulatowsta et el. 1990) medidos todos ellos sobre discurso narrativo elicitado.

\begin{tabular}{|c|c|c|}
\hline Nivel de análisis & Variable/rasgo del discurso & Parámetro empleado \\
\hline \multirow{13}{*}{ Intra-frase } & \multirow{4}{*}{ Elementos por categorías } & $\begin{array}{l}\text { Porcentaje de nombre/ verbo / adjetivo/ adverbio sobre total de } \\
\text { palabras }\end{array}$ \\
\hline & & Ratio nombre / verbo \\
\hline & & Ratio adjetivo/ nombre \\
\hline & & $\begin{array}{l}\text { Ratio pronombre/ concepto: } n .^{\circ} \text { de pronombres utilizados por el } \\
\text { sujeto para referirse a un concepto dado. }\end{array}$ \\
\hline & Productividad & Número total de unidades de contenido por narración \\
\hline & Corrección & Ratio de frases agramaticales por narración \\
\hline & \multirow{5}{*}{ Complejidad } & Número de cláusulas integradas en otras unidades \\
\hline & & $\begin{array}{l}\text { Número de oraciones subordinadas por unidad de contenido } \\
\text { (T - Unit) }\end{array}$ \\
\hline & & Número de oraciones subordinadas por narración \\
\hline & & $\begin{array}{l}\text { Longitud de la unidad de contenido: media de número de pala- } \\
\text { bras por unidad de contenido }\end{array}$ \\
\hline & & Número de palabras por unidad de contenido \\
\hline & \multirow{2}{*}{$\begin{array}{l}\text { Medidas de lexicalización: } \\
\text { se utiliza en estudios sobre } \\
\text { discurso narrativo licitado por } \\
\text { medio de una lámina en la } \\
\text { que se esperan respuestas de- } \\
\text { terminadas por parte de los } \\
\text { sujetos. }\end{array}$} & $\begin{array}{l}\text { Porcentaje de sujetos que verbalizan el término esperado para } \\
\text { un concepto dado }\end{array}$ \\
\hline & & $\begin{array}{l}\text { Número diferentes de términos usados por cada grupo para } \\
\text { expresar cada uno de los conceptos dados en la lámina. }\end{array}$ \\
\hline \multirow{7}{*}{$\begin{array}{l}\text { Inter-frase } \\
\text { Cohesión: medidos a } \\
\text { través del uso de ne- } \\
\text { xos inter - oraciona- } \\
\text { les }\end{array}$} & & Frecuencia de uso de nexos \\
\hline & & $\begin{array}{l}\text { Tipos de nexos empleados (medidos a través de clasificaciones a } \\
\text { priori) }\end{array}$ \\
\hline & & $\begin{array}{l}\text { Adecuación del uso de nexos: Porcentaje de nexos completos so- } \\
\text { bre el total de nexos }\end{array}$ \\
\hline & \multirow{4}{*}{ Tipología de errores } & Errores de repetición \\
\hline & & Errores de programación \\
\hline & & Errores de contradicción \\
\hline & & Errores de relación \\
\hline
\end{tabular}


Anexo 2. (Continuación)

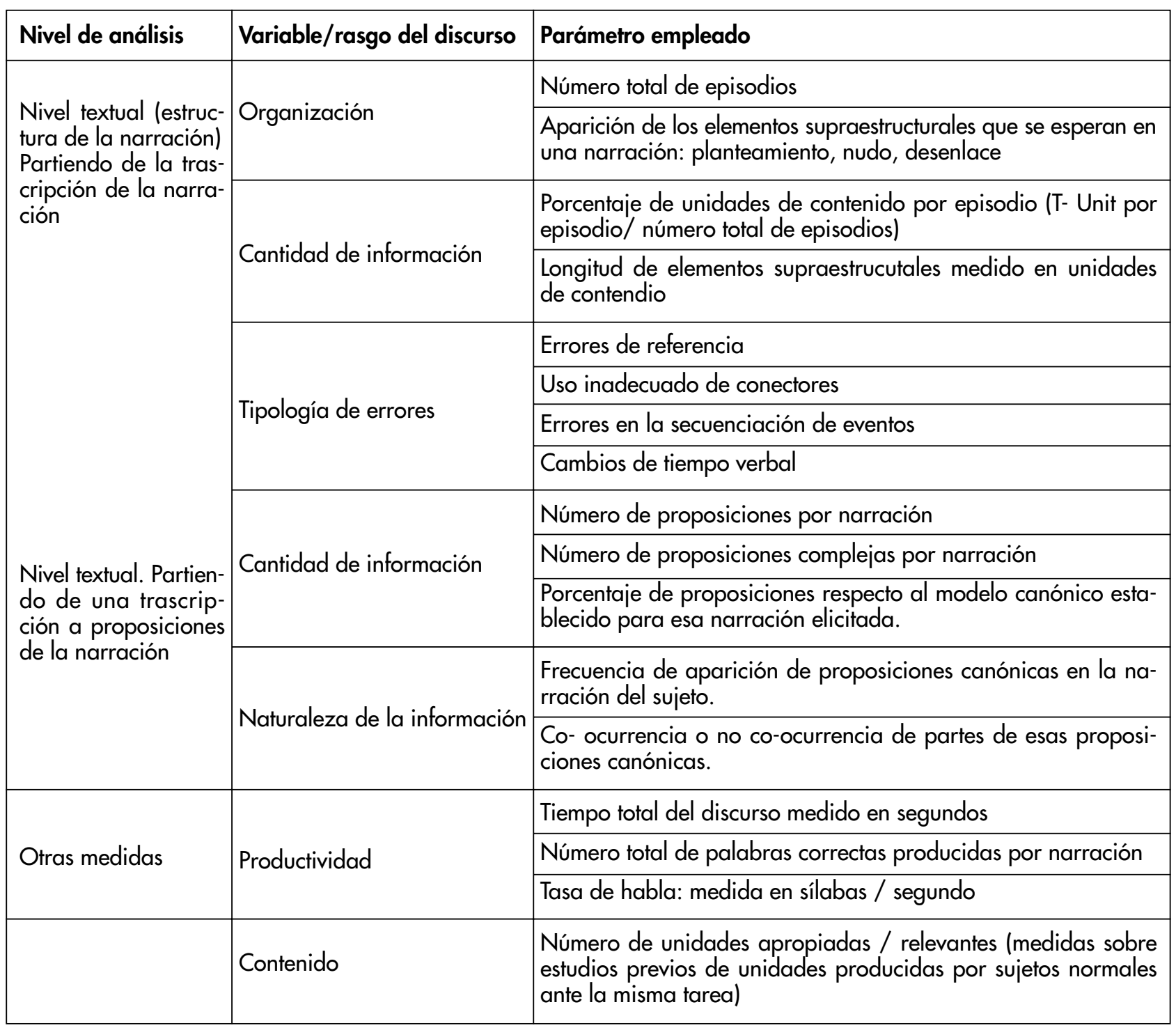

\section{Anexo 3. Parámetros medidos en el análisis del discurso para el caso presentado}

Parámetros para medir el habla entendida como conducta motora:

- Velocidad de habla: números de palabras ${ }^{1}$ por minuto.
- Bloqueo: Pausa o explosión anormal de un sonido al principio o dentro de la palabra.

- Alargamiento de sonido: Alargamiento de un fonema inicial, medio o final dentro de la palabra.

${ }^{1}$ Número de palabras: incluye todas la palabras de contenido y de función se omiten titubeos y falsos inicios, que se contabilizan a parte. Se computan fragmentos identificables como palabras fragmentadas, incorrectas, autocorrecciones, repeticiones, palabras de jerga, nexos oracionales y digresiones irrelevantes (veamos, pues..). 
Parámetros que se relacionan con la generación y la organización del discurso:

- Falsos inicios: Palabras o grupos de palabras inacabadas que posteriormente se completan o se sustituyen por otras. Ejemplo: «el pa... la madre».

- Autocorrecciones: Cambios de una palabra completa por otra que se ajusta mejor a lo que el sujeto quería decir. Ejemplo: "el el la niña» hay una repetición y una autocorrección (el por la).

- Repeticiones: número palabras o grupos de palabras repetidas, se contabiliza el número de repeticiones, por ejemplo «la la la niña» serían dos repeticiones de una sola palabra y "el perro el perro vino» sería una sola repetición de un grupo.

- Latiguillos: elementos semánticamente irrelevantes, que no amplían la informa- ción aportada por el discurso (veamos, pues, bueno, digamos...)

- Silencios: pausas inadecuadas dentro del discurso o adecuadas que exceden el tiempo que un oyente puede estimar habitual para continuar.

- Ratio nombre /verbo: número de nombres entre el número de verbos.

Parámetros que hacen referencia la concreción del lenguaje o a la eficacia del mismo

- Índice de eficiencia léxica: Número de palabras producido entre el número de unidades de contenido ${ }^{2}$.

- índice de adecuación: número de unidades de contenido correctas ${ }^{3}$ entre el número total de unidades de conten.

2 unidades de contenido: n. ${ }^{\circ}$ máximo de sintagmas (nominales, preposicionales, verbales y adjetivales) en que se puede subdividir el discurso, se incluyen los que forman parte de otro sintagma mayor. Ejemplo: «por el parque» contabilizaríamos dos sintagmas uno preposicional «por el parque» y uno nominal «el parque».

Esta definición no corresponde a la utilizada en la literatura anglosajona y se ha reformulado sobre la empelada en la adaptación del Perfil de Eficiencia Comunicativa.

3 unidades de contenido correctas: $n .^{\circ}$ de unidades de contenido tal y como se han definido previamente que se adecuan al contenido de la lámina con la que se ha elicitado el discurso y que no son repeticiones de ideas antes mencionadas. 